“C 2018 IEEE. Personal use of this material is permitted. Permission from IEEE must be obtained for all other uses, in any current or future media, including reprinting/republishing this material for advertising or promotional purposes, creating new collective works, for resale or redistribution to servers or lists, or reuse of any copyrighted component of this work in other works." 


\title{
A design and implementation of an ambulatory electrocardiogram (ECG) acquisition circuit for emergency application
}

\author{
Jiewei Feng ${ }^{1}$, Shahriar Hasan Shehab ${ }^{1}$, Yang Yang ${ }^{2}$, Nemai C.Karmakar ${ }^{1}$, Samir Gupta ${ }^{3}$ \\ 1. Department of Electrical and Computer Systems Engineering, Monash University \\ \{jiewei.feng1, shahriar.shehab, nemai.karmakar\}@monash.edu, \\ 2. School of Electrical and Data Engineering, University of Technology Sydney \\ yang.yang.au@ieee.org \\ 3. Department of Marketing, Monash University \\ samir.gupta@monash.edu
}

\begin{abstract}
This paper presents the design and development of an ECG data acquisition circuit for emergency applications. The ECG signal extraction method and the design of the analogue front-end circuit are discussed. This design has been implemented in a printed circuit board (PCB), with comparable size to a 50 cent Australian coin. By applying the testing approach with this prototype, the output ECG trace quality is overall satisfactory with a clear display of QRS complex and certain robustness to motion artifacts.
\end{abstract}

Keywords-Electrocardiogram(ECG); Motion Artifacts; Ambulatory ECG

\section{INTRODUCTION}

According to the statistics by ST Vincent's Hospital, 25\% of Australians have 3 or more risk factors for heart disease [1], which affects 1 in every 6 Australians, and causes 1 death in every 24 minutes [1]. Electrocardiogram (ECG) is a widely accepted tool for diagnosing and monitoring of heart disease [2]-[3]. An ECG is conventionally a medical test that monitors the electrical pulses generated from the heart muscle activity by using adhesive electrodes attached to the human body. The electrical activity is recorded and displayed in an electrocardiograph as a continuous waveform. As ECG has a regular pattern for a normal and healthy heart, any irregular heart rhythm or heart muscle damage can be reflected in the shape of the ECG signal, as a result of a change in the electrical activity of the heart. Therefore, heart problems could be diagnosed and monitored by analysing the pattern [1]-[2].

Although ECG is a widely used tool for health monitoring, there are some general problems with this medical test. Since the biological ECG signal from the human body is rather weak and susceptible to different types of noise, it generally suffers poor signal to noise ratio (SNR), especially due to the $50 / 60 \mathrm{~Hz}$ utility noise coupled by human body [4]. In the conventional ECG monitoring systems, the machine is too bulky for continuous everyday usage, and the cumbersome wires make the system inconvenient to use, even in a wearable Holter monitoring system [5].
To make ECG handy and comfortable for long-term monitoring, and to complement remote patient monitoring, ongoing research efforts have been put in the development of wearable ECG system and wireless body sensor network in the recent years. As a result, various convenient forms of ECG devices has evolved, some examples of which include Smart Vest [6], ECG monitoring shirt using dry electrodes with Planar-Fashionable Circuit Board (P-FCB) [5], contactless capacitive ECG monitoring system [7], and low power wireless ECG transducer employing amplitude modulation (AM) [8]. These developments of ECG system greatly enhance the convenience and the feasibility of continuous monitoring the health status of the end user.

Considering that ECG is one of the vital signals for an emergency patient with heart diseases, the applications of ECG can also be extended into ambulances and emergency department, these could provide opportunities for treatments to be planned and prepared before the patient arrives at the hospital [9]-[10]; as well as to facilitate the monitoring and diagnosis in the emergency department [11].

However, the application of ECG in the ambulance is still not highly developed in terms of system size, power consumption, multi-parameter functionality, and communication range. This work aims to develop a wireless ECG transducer for ambulance and emergency department. In this paper, the method for ECG signal extraction, and the design of a basic ECG signal acquisition circuitry are presented. The ECG circuit is comprised of 3 ECG electrode connections to form a Lead II configuration and noise cancelling with right leg drive. The susceptibility of ECG trace quality to motion artifacts has been highly reduced by this test approach and the developed ECG circuit. The rest of this paper is organised as follows: Section II presents circuit design and implementation, Section III explains the testing approach, Section IV discusses the results of testing and future plan, and the conclusion of this study comes in Section V. 


\section{CIRCUIT DESIGN AND IMPLEMENTATION}

\section{A. ECG Signals and System Diagram}

The ECG transducer generates a signal stream with amplitude from $50 \mu \mathrm{V}$ to $4 \mathrm{mV}$ with frequency components concentrating at 0.5 to $100 \mathrm{~Hz}$ [4]. Considering that the ECG signal is too weak and subjected to a variety of noises, the system in Fig. 1 has been designed to obtain a clear ECG waveform. The circuit design of the developed ECG data acquisition system in Fig. 2 is discussed block by block as follows:

\section{B. Circuit Design}

1) Radio Frequency Interference (RFI) Filter

Apart from the significant 50/60 Hz utility noise, the ECG signal is often exposed to strong radio frequency (RF) fields from the electrosurgery machines. Those machines generate strong fields with frequency from $500 \mathrm{kHz}$ to $3 \mathrm{MHz}$. To eliminate the RF interference, this design applied an $\mathrm{R}-\mathrm{C}$ low pass filter at the inputs of the Differential Amplifier Module [12], and this RFI filter could also provide an input bias path

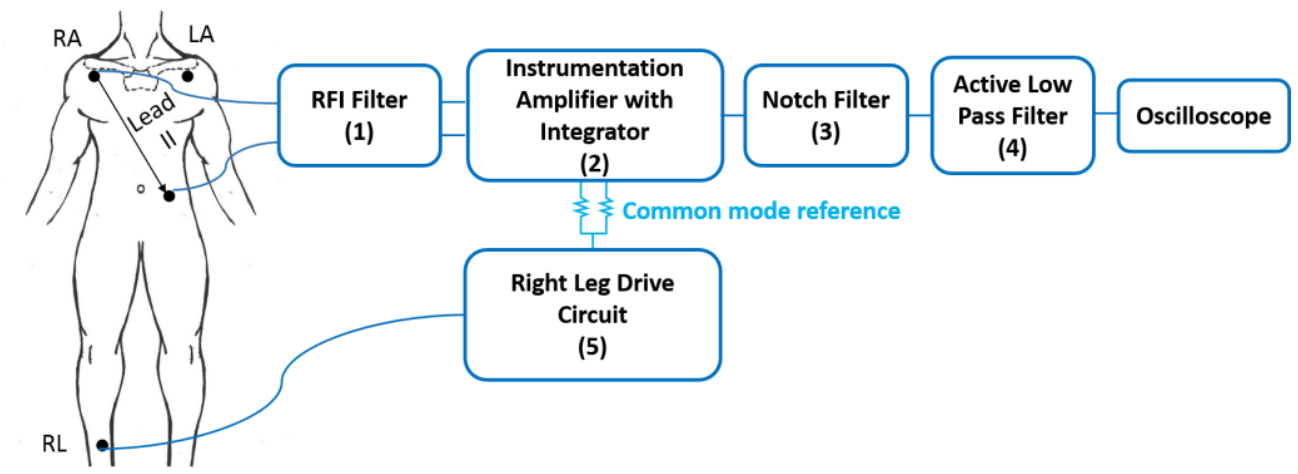

Fig. 1 Block diagram of the ECG acquisition circuit.

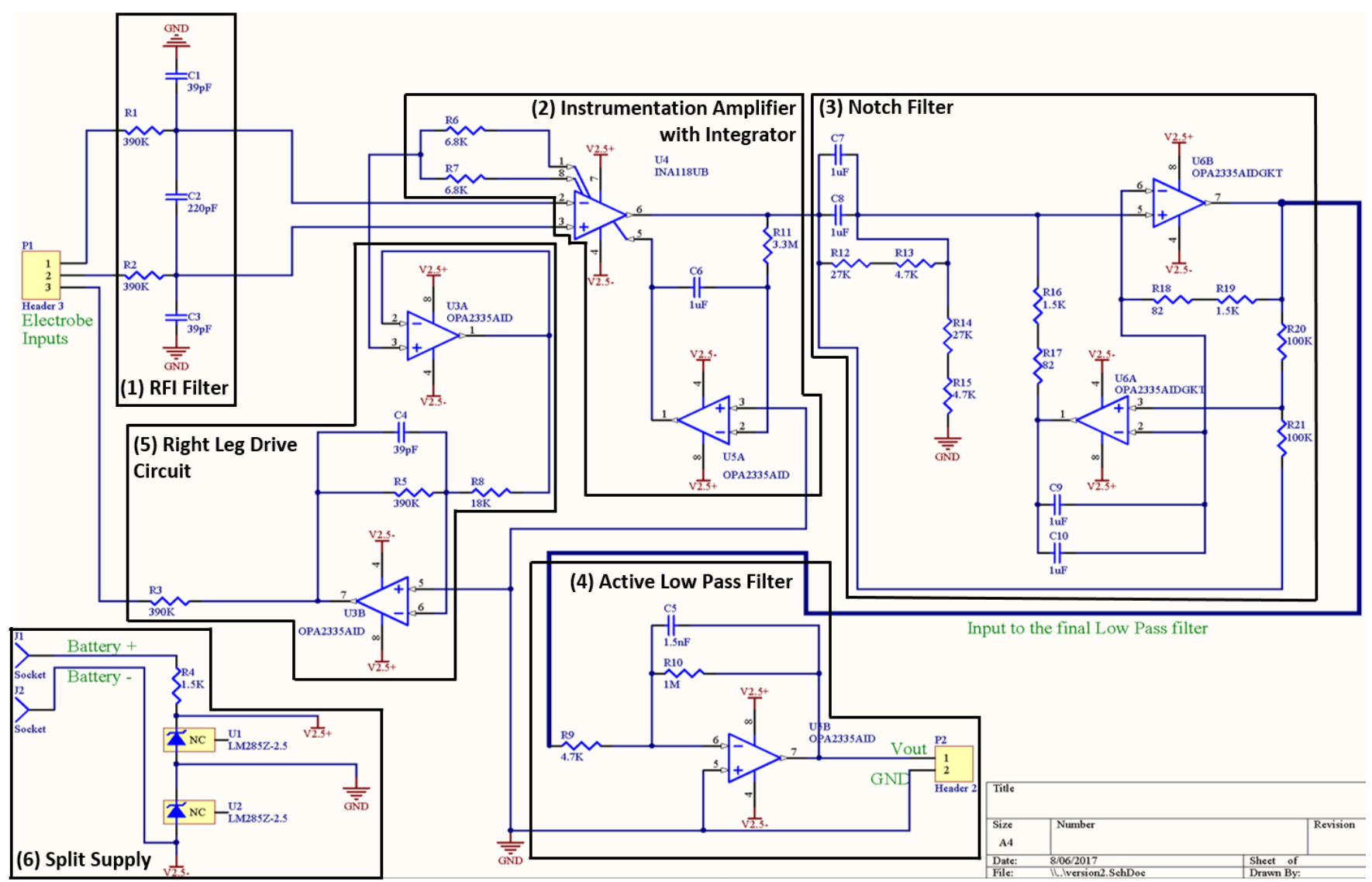

Fig. 2 Circuit diagram of the ECG analogue front-end. 
for the Differential Amplifier INA118 [13].

As it can be seen from Fig. 2, $R_{1}, R_{2}, C_{1}, C_{2}$ and $C_{3}$ form a bridge circuit across the inputs of the differential amplifier. In order to avoid reducing the high Common Mode Rejection Ratio (CMRR), the time constant of $R_{1} / C_{1}$ and $R_{2} / C_{3}$ should be identical to keep the bridge balanced [4].

$C_{2}$ connects between two inputs of the differential amplifier, and it is effectively connected in parallel with $C_{1}$ and $C_{3}$ that are grounded in series. As $C_{2}$ has much higher capacitance than $C_{1}$, it can significantly reduce any mismatch between the R-C filters formed at two input terminals [4].

Both differential and common mode signals would be the inputs of this filter. As these two types of signals will see different equivalent circuits, this results in two different bandwidths. Those are $B W_{\text {diff }}$ for differential input signal, and $B W_{c m}$ for common mode signal [4]. Suggested by [12], the cutoff frequency of the RFI filter shall not be lower than $100 \mathrm{~Hz}$. These two bandwidths can be determined as stated in [4]:

$$
\begin{aligned}
& B W_{\text {diff }}=\frac{1}{2 \pi R\left(2 C_{2}+C_{1}\right)} \\
& B W_{c m}=\frac{1}{2 \pi R C_{1}}
\end{aligned}
$$

\section{2) Instrumentation Amplifier with Integrator}

In order to extract the weak ECG signal with poor SNR, as well as to avoid amplifying noises, the differential amplifier plays a critical role in the ECG Signal Acquisition. The requirements for the differential amplifier are very high input impedance to minimise input current offset, very high CMRR to curb the common mode noise and increase SNR, low voltage offset drift to minimise unadjusted error, and low power consumption for battery operated systems [4], [6], [14].

The integrator circuit connects between the output and $V_{\text {ref }}$ of the instrumentation amplifier. By forming a feedback loop with low output impedance in the difference amplifier of INA 118 , it works equivalently as a high pass filter to remove the DC components (i.e. frequencies lower than $0.05 \mathrm{~Hz}$ in this case), while preserving a good CMRR of the instrumentation amplifier [13], [15]-[16].

\section{3) Notch Filter}

Given that the $50 \mathrm{~Hz}$ noise from main $\mathrm{AC}$ supply is right in the middle of the ECG bandwidth (i.e. $0.05-100 \mathrm{~Hz}$ ) [12], a notch filter together with right-leg drive circuit is included to eliminate this in-band interference.

By considering the difficulty of implementing a single op-amp Twin-T notch filter in the physical circuit, a Fliege notch filter is used for this design. This configuration only requires 4 precision components rather than 6 in the Twin-T filter, and slight mismatches of components can be more tolerated. Another advantage of this filter is that the Q and the centre frequency can be adjusted independently to each other [17], where $\mathrm{Q}$ is defined as the ratio between the $-3 \mathrm{~dB}$ bandwidth and centre frequency [12].

High precision components are necessary for a deep notch at $50 \mathrm{~Hz}$, a $45 \mathrm{~dB}$ Notch Depth is achievable by using components with tolerances $1 \%$ or fewer [11], and the best that could be hoped for is $40-50 \mathrm{~dB}$ with real-world components [17].

\section{4) Active Low Pass Filter}

The low pass filter in the final stage of the circuitry is to limit the signal bandwidth to $100 \mathrm{~Hz}$, as well as to increase the overall voltage gain to around 1000; therefore, an active low pass filter was designed.

Given that the voltage gain from the differential amplifier module is around 5, a voltage gain of 200 from the low pass filter would be sufficient for amplifying the ECG signal. As a result, a readable ECG trace would be expected at the output of this low pass filter

\section{5) Right Leg Drive Circuit}

As human body can act as an antenna, environmental noise can be easily coupled into a human, it is especially so with the $50 \mathrm{~Hz}$ utility noise, which is normally larger than the ECG biological signal in the human body. Right Leg Drive circuitry was used in this design to apply an amplified and inverted common-mode noise that feeds back to human. This can compensate the current in the human body and drive the common-mode voltage to a low value [4], [18]. As for the design, the common-mode signal is extracted from average of the two input signals to the instrumentation amplifier, and that is the mid-point of gain resistors $R_{G}$ in the instrumentation amplifier [13]-[14]. The output resistance $R_{16}$ is used for limiting the drive current that is flowing into human body via the electrodes [4].

6) Split Supply

Indicated from Fig. 2, two micropower voltage reference diodes are used to form the split supply to power the circuit, where the virtual ground is extracted from the middle point of diodes connection. The current limiting resistor is designed to provide sufficient current to the circuit, while to prevent the diodes from being damaged.

\section{Circuit Implementation}

The circuit design in Fig. 2 has been implemented as a printed circuit board (PCB) prototype after the validation of simulation and breadboard prototype. Surface mount components are adopted in the PCB design to minimise the size of the prototype, with the battery holder mounted at the back of the PCB. This prototype is in a circular shape with a diameter of $32 \mathrm{~mm}$. Its dimension is very similar to that of a 50 cent Australian coin, which has a diameter of $31.65 \mathrm{~mm}$ with the dodecagonal shape. Fig. 3 shows the comparison of dimensions of these two.

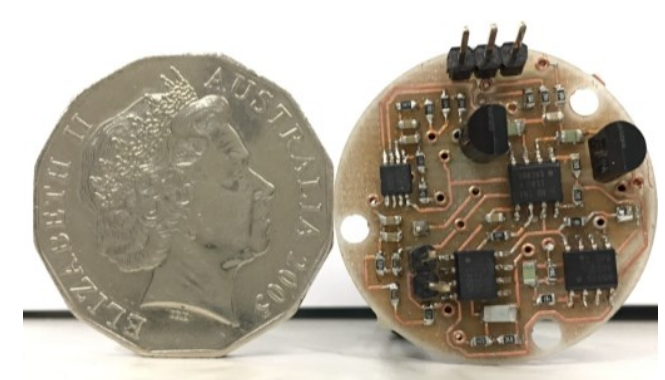

Fig. 3 Dimensions comparison between a 50 cents Australian coin and the ECG prototype 


\section{TEST APPROACH}

\section{A. Floating Electrodes}

In this design, $\mathrm{Ag} / \mathrm{AgCl}$ (floating) $3 \mathrm{M}^{\mathrm{TM}} \mathrm{Red} \operatorname{Dot}^{\mathrm{TM}}$ Electrodes will be used to pick up the electrical activities, which are generated from heart depolarisation and repolarisation. These conventional electrodes are not convenient for long duration application, as they may cause irritation and degradation of signal quality when the gel in the electrodes dries out after a long time usage [5]-[7]. However, considering the proposed application of this design is for a short-duration usage in ambulances/emergency applications, which demands consistent good trace quality, these floating electrode may be more suitable in this regard, as compared to the developed dry electrodes. This is due to that the electrical double layer formed by the metal-electrolyte interface is floated between the snap and gel sponge, rather than directly between metal and skin as in the dry electrodes; the noise associated with motion could be suppressed by a factor of 10 , thus improving the stability [19]-[21].

\section{B. Leads Configuration and Electrodes Placement}

Indicated by Fig. 1, this design utilises a 3-lead configuration. In this configuration, the bipolar leads work in a similar fashion to a voltage meter to pick up the electrical signal generated from heart activities. As the main travel direction of the electrical signal is left, inferior and posterolateral [22], which is very similar to that of Lead II directional potential measurement, Lead II would be a good candidate to acquire the principle waves of ECG signal.

Suggested by [19], [23], the electrodes placed at the periphery of limbs (i.e. standard locations) would lead to the records being highly susceptible to muscle noise, even from a single finger movement. Placing electrodes at locations RA, LA and LL in Fig. 1 would be able to reduce the susceptibility to muscle noise, while providing a good quality of ECG trace as in the standard positions. Suggested by the name "Right leg drive", the inverted feedback signal from the right leg drive circuit is returned to the human body via the electrode attached to location RL in Fig. 1.

\section{Skin Preparation}

The "barrier" layer Stratum Carenum is the outermost layer of skin. It stores dead cells and has high impedance (i.e. typically $50 \mathrm{k} \Omega / \mathrm{cm}^{2}$ ), which is believed to be the major problem source for ECG signal quality. Skin deformation is the major source of motion artifacts, and this could cause a change of $5 \mathrm{mV}$ in the skin potential between the inside and outside of the "barrier" layer of the skin [20]. By applying fine sand paper with around 20 strokes, part of the Stratum Carenum will be removed, and this results in a reduction of this change in skin potential to a negligible level and a significant drop of skin impedance to less than $5 \mathrm{k} \Omega / \mathrm{cm}^{2}$ [20], [24]. The drop of skin impedance also reduces the disturbance of $50 \mathrm{~Hz}$ utility interference, static electricity and conversion of common-mode voltage to differential voltage, where the last disturbance is due to the impedance variation in different locations of human body [20]. In this test, prior to placing electrodes, 3M $\mathrm{M}^{\mathrm{TM}}$ Red Dot ${ }^{\mathrm{TM}}$ Trace Prep was used by mild skin abrasion to reduce skin impedance and hence the disturbance.

\section{RESULTS AND DISCUSSION}

\section{A. Test results}

Fig. 4 shows the prototype test result while the subject is standing. The principal components (i.e. P wave, QRS complex, and $\mathrm{T}$ wave) can be observed on the oscilloscope with negligible noise disturbance. This implies that the ECG signal extraction and noise filtering of the circuit are functioning properly; the $V_{\text {peak-peak }}$ of the QRS complex is measured to be $2.2 \mathrm{~V}$, which implies sufficient amplification has been provided by the circuit.

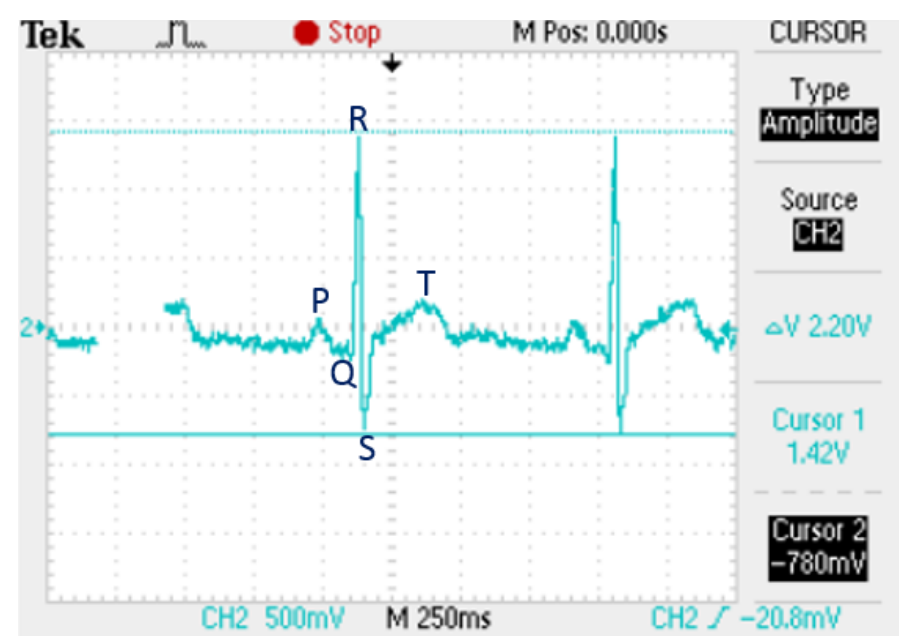

Fig. 4 Prototype test result while the subject is standing.

Fig. 5 show the sensitiveness of motion artifacts to the ECG trace quality. Fig. 5(a) shows the ECG waveform with finger motion, and only minor distortion to all the principle waves could be observed. Fig. 5(b) shows the ECG trace with walking motion, and it could be observed that $\mathrm{P}$ wave is totally masked, while QRS complex and $\mathrm{T}$ wave are still readable; the spurious peaks between the QRS complexes may be caused by the poor snap - crocodile clip connection. Therefore, it could be concluded that certain robustness has been obtained by the circuit prototype and test method.

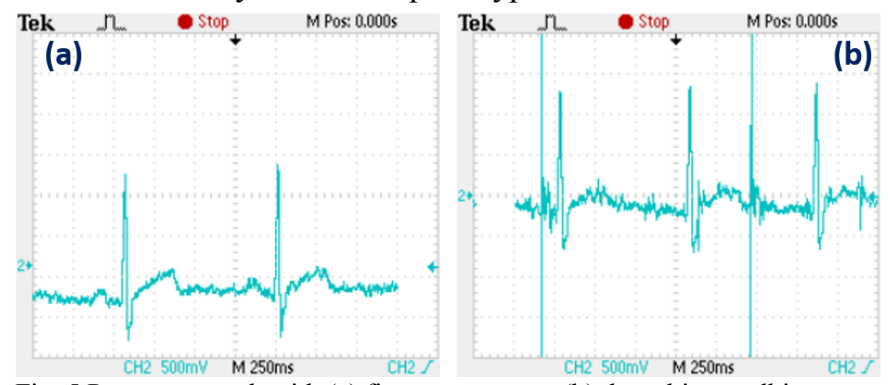

Fig. 5 Prototype result with (a) finger movement (b) the subject walking.

\section{B. Future plan}

At this stage, the ECG circuit design has not yet been fully finalised. As discussed in the Test results section, the poor 
snap-crocodile connection may hinder an accurate acquisition of ECG signal; snap type ECG cables with shielding layer will be utilised to replace the banana cable. Shield guarding circuity will be adopted to the circuit design to further reduce the level of interference coupled to the circuit [25].

Fig. 6 shows the proposed system design for ambulance/emergency applications. The bottom layer consists of an $\mathrm{Ag} / \mathrm{AgCl}$ (floating) electrode. This electrode and hence the whole system will be attached to the human body such that the lengths of wire connections to the rest two electrodes are minimised. The finalised ECG acquisition circuit will be placed in the middle layer, with batteries affixed to its backside. The top layer will be the microcontroller unit (MCU) for signal processing and data packaging, and a wireless module for transmitting the ECG signal in digital form. Through the medium of the built-in wireless receiver of consumer electronics (e.g. IPad, laptop, mobile phones), this digital signal can be plotted into real-time ECG trace via a graphical user interface (GUI). By this means, the real-time ECG signal could be monitored in a more flexible manner, as compared to utilising dedicated medical instruments (e.g. electrocardiography). This could facilitate monitoring the health status of patients, and hence prepare timely treatments in the emergency room and first aid in ambulances.

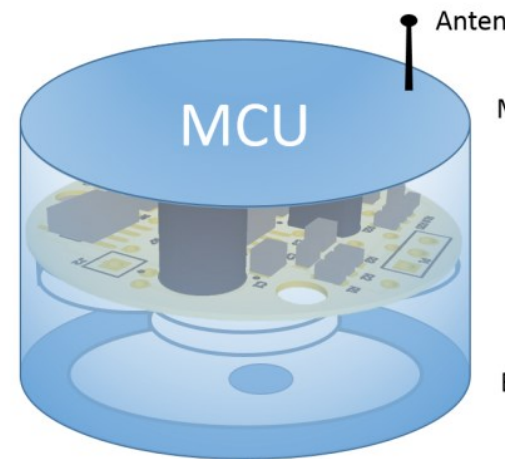

Micro controller unit (MCU) and wireless module

\section{ECG circuit layer} and batteries

ECG silver dot bottom layer

Fig. 6 Proposed ECG wireless transducer system design.

\section{CONCLUSIONS}

In this paper, the testing approach for reducing motion artifacts, and the design of a basic 3 lead ECG acquisition circuit have been presented. Satisfactory ECG trace quality has been obtained in the developed ECG prototype, and distortion by motion artifacts has been highly reduced. The circuit design has not yet been fully finalised at this stage, and this work will be expanded for ambulance/emergency applications with the proposed future plan.

\section{ACKNOWLEDGMENT}

This work was supported by the supervision and guidance from Dr Abdur Rahim, as well as resources from Monash University and Monash Microwave, Antennas, RFID and Sensors (MMARS) Laboratories.

\section{REFERENCES}

[1] "ECG," St Vincent's Hospital Heart Healt, [Online]. Available: https://svhhearthealth.com.au/Procedures/Tests/ECG. [Accessed 2003 2017].
[2] "ECG test," Better Health, 2 2017. [Online]. Available: https://www.betterhealth.vic.gov.au/health/conditionsandtreatments/ecgtest. [Accessed 2003 2017].

[3] S. B. M. T. I. a. N. K. D. A. M. Jobayer, "Design and implementation of 3-lead ECG machine for rural medification," in 2015 International Conference on Electrical Engineering and Information Communication Technology (ICEEICT), Dhaka, 2015.

[4] Y. Li, X. Xu, Y. Liu and Y. Tian, "Analysis and Implement of ECG Front-End Signal Processing Circuit," in 2011 International Conference of Information Technology, Computer Engineering and Management Sciences, 2011.

[5] J. Yoo, J. Long Yan, J. Seulki Lee, J. Hyejung Kim and J. Hoi-Jun Yoo, "A Wearable ECG Acquisition System With Compact PlanarFashionable Circuit Board-Based Shirt," IEEE Transactions on Information Technology in Biomedicine, vol. 13(6), pp. 897-902, 2017.

[6] P. Pandian, K. Mohanavelu, K. Safeer, T. Kotresh, D. Shakunthala, P. Gopal and V. Padaki, "Smart Vest: Wearable multi-parameter remote physiological monitoring system," Medical Engineering \& Physics, vol. 30(4), pp. 466-477, 2008.

[7] E. Nemati, M. J. Deen and T. Mondal, “A wireless wearable ECG sensor for long-term applications," IEEE Communications Magazine, vol. 50(1), pp. 36-43, 2012.

[8] Y. Yang, X. Zhu, K. Ma, R. B. V. B. Simorangkir, N. Karmakar and K. P. Esselle, "Development of Wireless Transducer for Real-Time Remote Patient Monitoring," IEEE Sensors Journal, vol. 16, no. 12, pp. 46694670, Jun. 2016.

[9] C. Zywietz, V. Mertins, D. Assanelli and C. Malossi, "Digital ECG transmission from ambulance cars with application of the European Standard Communications Protocol SCP-ECG," in Computers in Cardiology 1994, 1994.

[10] K. Suma K V, K. Sandeep S, K. Vikram S, K. Hanjar and K. Sudharshan S M, "Cardiogenic shock monitoring system for ambulance," in 2015 International Conference on Advances in Computing, Communications and Informatics, 2015.

[11] N. C. Karmakar, Y. Yang and A. Rahim, "Microwave Sleep Apnoea Monitoring," Springer, Singapore, 2018, DOI: 10.1007/978-981-106901-7.

[12] J. E. I. I. \&. S. Carr, The technician's EMI handbook clues and solutions, Boston: Boston : Newnes, 2000 .

[13] "INA118 - Texas Instruments," 01 2016. [Online]. Available: http://www.ti.com/lit/ds/symlink/ina118.pdf. [Accessed 1503 2017].

[14] M. W. Hann, "Ultra Low Power, 18 bit Precision ECG Data Acquisition System," $62013 . \quad$ [Online]. Available: http://www.ti.com/lit/ug/slau516/slau516.pdf. [Accessed 2703 2017].

[15] Y. Yang, Radio Frequency-Based Wireless Monitoring of Sleep Apnoea Patient, Ph.D thesis, http://arrow.monash.edu.au/hdl/1959.1/859040.

[16] T. Kugelstadt, "Getting the most out of your instrementation amplifier design," $2005 . \quad$ [Online]. Available: http://www.ti.com/lit/an/slyt226/slyt226.pdf. [Accessed 0705 2017].

[17] B. Carter, "High-speed notch filters," Analog Applications Journal, pp. 19-25, 2006.

[18] A. Wong, A. Kong-Pang Pun, A. Yuan-Ting Zhang and A. Chiu-Sing Choy, "An ECG measurement IC using driven-right-leg circuit," in 2006 IEEE International Symposium on Circuits and Systems 2006, 2006.

[19] J. Webster, "Interference And Motion Artifact In Biopotentials," in IEEE 1977 Region Six Conference Record, 1977, 0 1977, pp.53-64, 1977.

[20] J. G. Webster, "Reducing Motion Artifacts and Interference in Biopotential Recording," IEEE Transactions on Biomedical Engineering, Vols. BME-31(12), pp. 823-826, 1984.

[21] H. Tam and J. G. Webster, "Minimizing Electrode Motion Artifact by Skin Abrasion," IIEEE Transactions on Biomedical Engineering, Vols. Vol.BME-24(2), pp. 134-139, March 1977.

[22] a. S. B. a. R. Stroobandt (Roland) and A. F. S. author., "The Normal ECG and the Frontal Plane QRS Axis," in ECG from basics to essentials : step by step, Chichester, West Sussex, UK ; Hoboken, NJ : Wiley Blackwell , 2015, pp. 58-59. 
[23] R. E. Mason and I. Likar, "A new system of multiple-lead exercise electrocardiography," American heart journal, vol. Vol.71, pp. 196-205, 1966.

[24] C. D. Oster, "Proper Skin Prep Helps Ensure ECG Trace Quality," [Online]. Available: http://multimedia.3m.com/mws/media/358372O/proper-skin-prep-ecgtrace-quality-white-paper.pdf. [Accessed 0305 2017].

[25] A. P. C. A. G. A. C. MettingVanRijn, "High-quality recording of bioelectric events. Part 1. Interference reduction, theory and practice," 9 1990. [Online]. Available: https://www.biosemi.com/publications/pdf/Interference reduction.pdf. [Accessed 1605 2017]. 